\title{
The effectiveness of the oncolytic activity induced by Ad5/F35 adenoviral vector is dependent on the cumulative cellular conditions of survival and autophagy
}

\author{
SO Y. KIM ${ }^{1,2}$, SUJIN KANG ${ }^{1,2}$, JAE J. SONG ${ }^{1}$ and JOO-HANG KIM ${ }^{3}$ \\ ${ }^{1}$ Institute for Cancer Research, ${ }^{2}$ Brain Korea 21 Project for Medical Science, \\ ${ }^{3}$ Department of Internal Medicine, College of Medicine, Yonsei University, Seoul, Republic of Korea
}

Received November 21, 2012; Accepted January 22, 2013

DOI: $10.3892 /$ ijo.2013.1812

\begin{abstract}
To overcome the poor tumor transduction efficiency of adenovirus serotype 5 (Ad5) observed in several types of cancer, the fiber region of Ad5, apart from its tail, was replaced by adenovirus serotype 35 (Ad35). The chimeric Ad5/F35 adenoviral vector did not exhibit any significant enhancement of transduction efficiency. CD46, a receptor for Ad35, was expressed in relatively small amounts in most of the cancer cells examined. Therefore, we investigated the pivotal factor(s) that render cancer cells susceptible to transduction. We discovered that the tumor transduction efficiency of Ad5/F35 was enhanced in the presence of rapamycin, an autophagy inducer, in some cancer cells. Analysis of survival potential and cell proliferation rates revealed that $\mathrm{Ad} 5 / \mathrm{F} 35$ exerted a more pronounced oncolytic effect in cancer cells with higher survival potential in the presence of rapamycin.
\end{abstract}

\section{Introduction}

Adenoviral vectors are frequently used for gene transfer, in spite of their shortcomings, such as increased immunogenicity (which may occasionally prove beneficial in cancer treatment), prevalence of pre-existing anti-Ad immunity and lack of specific

Correspondence to: Professor Jae J. Song, Institute for Cancer Research, College of Medicine, Yonsei University, 50 Yonsei-ro, Seodaemun-gu, Seoul 120-752, Republic of Korea

E-mail: jjs109@yuhs.ac

Professor Joo-Hang Kim, Department of Internal Medicine, College of Medicine, Yonsei University, 50 Yonsei-ro, Seodaemun-gu, Seoul 120-752, Republic of Korea

E-mail:kjhang@yuhs.ac

Abbreviations: CAR, coxsackievirus and adenovirus receptor; ITR, inverted terminal repeats; GFP, green fluorescent protein; FITC, fluorescein isothiocyanate; APC, allophycocyanin; ADP, adenovirus death protein; GOPC, Golgi-associated PDZ and coiled-coil motif-containing protein; $\mathrm{LDH}$, lactate dehydrogenase

Key words: oncolytic activity, autophagy, Ad5/F35, CD46, rapamycin targeting. However, they also possess significant advantages, such as efficient transgene delivery, expression in both dividing and non-dividing cells and ease of propagation to high titers (1-3).

Although adenoviral vectors offer these advantages, poor tumor transduction efficiency in several types of tumor, dense stromal tissue impeding intratumoral virus spread and immunemediated viral clearance, remain the key factors limiting cancer gene therapy and virotherapy $(1,4,5)$. Over a number of years, extensive research has been directed toward overcoming these limitations. The reason for the poor transduction of adenoviral vectors is that tumor cells exhibit limited surface expression of the adenovirus 5 (Ad5) receptors, specifically the coxsackievirus and adenovirus receptor (CAR), $\alpha_{\mathrm{v}} \beta_{3}$ and $\alpha_{\mathrm{v}} \beta_{5}$ integrins. Therefore, numerous studies have focused on the modification of the adenoviral fiber region that binds to the receptors of tumor cells, to facilitate an eventual efficient infection. The structure of the fiber can be divided into three domains: an N-terminal tail, that attaches to the penton base, a central shaft with repeating motifs of $\sim 15$ residues and a C-terminal globular 'knob' domain, that functions as the cellular attachment site (6). The fiber knob has a central depression and three symmetry-related valleys, initially considered to be the binding sites for CAR (7). The first step involving the binding of the virus to the CAR receptor of target cells occurs via the $\mathrm{C}$-terminal knob domain of the fiber protein. The second step is the interaction of Arg-Gly-Asp (RGD) motifs in the penton base with $\alpha_{v} \beta_{3}$ and $\alpha_{v} \beta_{5}$ integrins, which are the secondary host cell receptors, facilitating the internalization of virus via receptormediated endocytosis. However, Ad5-mediated gene transfer is inefficient in a number of tissues, such as endothelial $(8,9)$, smooth muscle (8), differentiated airway epithelial (10) and brain tissue (11), as well as peripheral blood cells (12), due to the lack of corresponding primary and/or secondary receptors.

Several studies have been published, regarding the direct genetic modification of adenoviral particles as a strategy to increase the infection efficiency in specific target cells (13-16). These studies have demonstrated that the fiber region of adenovirus serotype 35 (Ad35), which possesses shorter fiber proteins compared to Ad5, exhibits a different pattern of tropism. Therefore, it has been utilized to construct a chimeric form with the fiber region of Ad5, with the expectation of higher transduction efficiency in cancer cells $(13,17)$. Since CD46 is 
a receptor for Ad35 that is ubiquitously expressed in human cells (18), chimeric Ad5/F35 fiber proteins may be expected to transduce in various human cancer cell lines more effectively, including CAR-negative cell lines. Several tumor cell lines, including melanoma, require high adenovirus doses for sufficient gene expression, due to the lower expression of CAR (19). Therefore, the Ad5/F35 chimeric vector may be a promising candidate for efficient gene transfer into human cancer cell lines. Toyoda et al (20) reported that the adenovirus with chimeric Ad5/F35 fiber proteins exhibited enhanced transfection efficiency in human pancreatic cancer cells. However, $\mathrm{Yu}$ et al $(21,22)$ demonstrated that the transduction efficiency of Ad5/F35 did not directly correlate with the expression level of CD46. In addition to the distinct cell entry pathways of Ad35, another advantage is the ability of the vector to induce autophagy through the CD46-Cyt-1/GOPC pathway, via the autophagosome formation complex, Vps34/Beclin-1 (18), which ultimately leads to viral replication and oncolysis (23).

Autophagy is a regulated process for the degradation of cellular components, that has been well-conserved in eukaryotic cells. Autophagy can be activated in response to various physiological and pathological stimuli to promote cell survival, or to act as a mode of cell death (24). Among the genes/proteins that regulate autophagy, mTOR, which belongs to the phosphatidylinositol kinase-related protein kinase family and has been identified as the cellular target of rapamycin, appears to play an important role (25). mTOR is not only involved in a nutrient-sensitive signaling pathway and autophagy, but also modulates apoptotic pathways (26). The modulation of mTOR activity, however, may have pro- as well as anti-apoptotic effects, since rapamycin, depending on the context, may exert both pro- and anti-apoptotic effects $(27,28)$.

To overcome the non-specific transcription of adenoviral vectors, conditional replication of the adenovirus and specific targeting of tumor cells have been used (2). Briefly, these efforts can be categorized into two general approaches. The first one is deleting the adenoviral genes that are essential for viral replication in normal cells, but are dispensable in tumor cells (29). The second approach is selectively limiting the expression of the adenoviral genes that are essential for viral replication in specific types of tumors or tissues, by using tumor- or tissue-specific promoters (29-31). In detail, among various tumor-specific promoters, the survivin promoter has been proven to be a promising candidate for its application in transcriptional targeting of adenoviral vector-based cancer gene therapy or oncolysis for melanoma $(32,33)$.

In the present study, we evaluated the effect of cytomegalovirus (CMV) promoter-driven oncolytic Ad5/F35 vector on various cancer cells. Our results suggest that the oncolytic activity induced by Ad5/F35 specifically correlates with the survival potential of the cell types, irrespective of their doubling time, which renders these cancer cells more sensitive to the autophagy inducer.

\section{Materials and methods}

Cell culture. The human cancer cell lines DU145 (prostate adenocarcinoma), MDA-MB-231 (breast adenocarcinoma), A549 (lung adenocarcinoma), HCT116 (colon carcinoma) and A375 (skin melanoma), were cultured in Dulbecco's modi- fied Eagle's medium (DMEM) with $10 \%$ fetal bovine serum (FBS) (HyClone, Logan, UT, USA). U373-MG, U87-MG, U251N and U343 (human malignant glioma), Jurkat (human T cell leukemia), SK-MEL-2 and SK-MEL-3 (human skin melanoma) cells were cultured in RPMI-1640 with 10\% FBS. HeLa (human cervical adenocarcinoma) cells were cultured in minimal essential medium (MEM) with 10\% FBS. HEM (human epidermal melanocyte) cells were cultured in melanocyte growth medium containing low levels of serum and fully supplemented with growth factors and antibiotics. 293A cells (a subclone of the 293 human embryonic kidney cell line) were cultured in DMEM with 10\% FBS (HyClone). Cells were maintained in a $37^{\circ} \mathrm{C}$ humidified atmosphere containing $5 \% \mathrm{CO}_{2}$.

Reagents and antibodies. Anti-mTOR, anti-phospho-Akt, anti-Bcl-2, anti-Bcl-xL, anti-LC3B antibodies and wortmannin were purchased from Cell Signaling Technology (Beverly, MA, USA). Fluorescein isothiocyanate (FITC)-conjugated anti-CD46, allophycocyanin (APC)-conjugated anti-mouse IgG1 and mouse IgG fluorescence control isotype antibodies were purchased from BD Biosciences (San Jose, CA, USA). Anti-CAR and anti-actin antibodies, 3-methyladenine (3-MA) and rapamycin were purchased from Santa Cruz Biotechnology (Santa Cruz, CA, USA). All other chemicals, including pepstatin A, E-64d and curcumin were purchased from SigmaAldrich (St. Louis, MO, USA).

Construction of chimeric adenoviral vectors. For the construction of chimeric sequences of Ad5 and Ad35 fiber regions, a synthetic gene (2374 bp) encoding Ad5 and Ad35 fibers was cloned into XbaI/KpnI-digested pBluescript II SK(-) and named pBSK[2374]. DNA synthesis was carried out by Epoch Biolabs (Sugar Land, TX, USA). The synthesized DNA sequences spanned a region from the 30470th (XbaI) to the 33599th $(K p n I)$ nucleotide of the human Ad5 genome sequence, with substitution of the Ad35 fiber shaft and knob domain. To remove the KpnI site present within the Ad35 shaft, the nucleotide sequence ggtacc was replaced with the sequence $g g c a c c$, without altering the amino acid sequence. The resultant sequences were a mixture of the Ad5 fiber tail domain and the Ad35 fiber shaft and knob domain. Following the digestion of pBSK[2374] with $X b a \mathrm{I} / K p n I$, the insert was subcloned into $X b a \mathrm{I} / K p n \mathrm{I}$-digested pSK[5543], a structure that has been previously described in detail (34). The resultant product was used as a shuttle vector. This newly constructed adenovirus shuttle vector with chimeric fibers, pSK [5543 with 5/35 fiber], was digested with $X m n I$ and the adenoviral vector vmdl324Bst containing the Ad5 genome lacking the E1 region (340-4640 in the nucleotide sequence of Ad5) and the E3 region (28592-30470 in the nucleotide sequence of Ad5) and the IX gene was linearized with SpeI for homologous DNA recombination in the $E$. coli strain, BJ5183. To generate Ad5/F35 chimeric fiber-incorporated green fluorescent protein (GFP)-expressing adenovirus, further homologous recombination was performed after subcloning the GFP gene from pEGFP-N1 ( $A f$ III-blunt-BamHI) into the pSP72- $\Delta \mathrm{E} 3-$ adenovirus death protein (ADP) Ad shuttle vector (35), after digestion with KpnI-blunt-BamHI for substitution of ADP with GFP. Subsequently, the second homologous recombination was performed in BJ5183 following digestion of the shuttle vector 
with $X m n I$ and of the adenoviral vector with $\operatorname{SrfI}$. Finally, pCA14 containing the IX gene, which plays a role in efficient viral proliferation, was linearized with $X m n I$ for homologous DNA recombination with the previous recombinant adenoviral vectors following digestion with $B s t \mathrm{BI}$. To verify the respective homologous recombinants, plasmid DNA was purified from an E. coli culture grown overnight, digested with HindIII and the digestion pattern was analyzed. The appropriate homologous recombinant adenoviral plasmid DNA was digested with PacI and transfected into 293A cells to generate GFP-expressing chimeric adenoviruses.

These chimeric adenoviruses, with or without the GFP gene, were propagated in 293A cells and amplified for purification, according to standard methods. Titration was performed by estimating the infectious viral particles with a standard plaque assay kit developed by Qbiogene (Carlsbad, CA, USA) in 293A cells. The Adeno-X Rapid Titer kit (Clontech; Mountain View, CA, USA) was used for the titration of GFP-expressing adenovirus.

Construction of oncolytic adenoviral vectors. For the construction of adenoviral vectors, a synthetic gene (3484 bp) was used as a backbone construct for the shuttle vector and the DNA was named pBSK[3484]. It consisted of inverted terminal repeats (ITR) - packaging signal - mouse survivin promoter-E1A-BGH polyA-E1B and 55-kDa gene cassetteAd E1 right region (in the order listed). The mouse survivin promoter may be replaced with other promoter types (CMV promoter, human survivin promoter), through digestion with $K p n I$ and $X h o I$, respectively, for the examination of promoter activity. pcDNA3.1/Hygro was used as a template for PCR amplification of the CMV promoter, with the sense primer, 5'-CGGGGTACCGATGTACGGGCCAGAT-3', and the antisense primer, 5'-CCGCTCGAGAATTTCGATAAGCCAG-3'. Following digestion of the PCR product of the CMV promoter with $K p n \mathrm{I} / X h o \mathrm{I}$, it was inserted into $K p n \mathrm{I} / \mathrm{XhoI}$-digested pBSK[3484]. Human survivin promoter was synthesized from +1 to -268 nucleotides of the survivin promoter and then inserted into KpnI/XhoI-digested pBSK[3484]. For the replacement of the $\mathrm{CMV}$ promoter or the human survivin promoter, the E1B 55-kDa gene cassette was deleted by EcoRI and SalI digestion, followed by blunting, so as to provide oncolytic activity. These various types of pSK[3484] constructs were again digested with FspI and BamHI and ligated into SspI and BglII-digested pCA14 for the formation of the final E1 shuttle vector. The pCA14-[3484] shuttle vector was linearized by XmnI digestion. The linearized pCA14[3484] was co-transformed into E. coli BJ5183, together with Bst $\mathrm{BI}$-digested vmdl324Bst, for homologous recombination. To verify the respective homologous recombinants, the plasmid DNA, purified from an overnight-grown E. coli culture, was digested with HindIII and the digestion pattern was analyzed. The homologous recombinant adenoviral plasmid DNA was digested with $\mathrm{PacI}$ and transfected into 293A cells to generate tumor-selective, replication-competent Ad-3484-CMV- $\Delta$ E1B-derived $\Delta$ E1B19/55 or Ad-3484-human survivin promoter-derived $\Delta \mathrm{E} 1 \mathrm{~B} 19$.

Flow cytometric analysis. Cancer cell surface receptors (CAR and CD46) were quantified by flow cytometric analysis. After various cancer cells were trypsinized and washed twice with ice-cold PBS, they were separately incubated with mouse anti-CAR antibody (Santa Cruz Biotechnology) for $1 \mathrm{~h}$ at $4^{\circ} \mathrm{C}$. After washing twice with ice-cold PBS, cells were incubated with APC-conjugated anti-mouse $\mathrm{IgG}$ antibody for $45 \mathrm{~min}$ at $4^{\circ} \mathrm{C}$ in the dark. Subsequently, the cells were washed twice with ice-cold PBS, incubated again with FITC-conjugated anti-CD46 for $1 \mathrm{~h}$ at $4^{\circ} \mathrm{C}$ in the dark, then washed twice with ice-cold PBS. Mouse IgG fluorescence control antibody was used as the negative control. Finally, the cells were suspended again in PBS and analyzed using a FACS Calibur flow cytometer (BD Biosciences, Lincoln Park, NJ, USA).

Cytopathic effect (CPE) assay. To evaluate the CPE of replication-competent adenoviruses, cells were first plated to $\sim 80 \%$ confluency into 24- or 48-well plates. They were pre-treated with 3-MA (Santa Cruz Biotechnology), wortmannin (Cell Signaling Technology), or rapamycin (Santa Cruz Biotechnology) for $1 \mathrm{~h}$ and were then infected with replication-competent adenoviruses of various multiplicities of infection (MOI). After $8 \mathrm{~h}$ of infection, cells were monitored daily under a microscope. When the infected cells exhibited cell lysis at the lowest MOI, the remaining cells on the plate were fixed with $4 \%$ paraformaldehyde and stained with $0.5 \%$ crystal violet.

Protein extracts and polyacrylamide gel electrophoresis. Cells were lysed with 1X Laemmli lysis buffer [62.5 mM Tris (pH 6.8), 2\% sodium dodecyl sulfate (SDS), 10\% glycerol, $0.002 \%$ bromophenol blue] and boiled for $10 \mathrm{~min}$. Protein content was measured using BCA Protein Assay reagent (Pierce, Rockford, IL, USA). The samples were diluted with $1 \mathrm{X}$ lysis buffer and $\beta$-mercaptoethanol was added to a concentration of $350 \mathrm{mM}$. Equal amounts of protein were loaded onto $10 \%$ SDS polyacrylamide gels. SDS-polyacrylamide gel electrophoresis (PAGE) analysis was performed according to the procedure of Laemmli using a Hoefer gel apparatus.

Immunoblot analysis. Proteins were separated by SDS-PAGE and electrophoretically transferred onto polyvinylidene fluoride (PVDF) membranes. Each PVDF membrane was blocked with 5\% non-fat dry milk in PBS-Tween-20 (0.1\%, v/v) at room temperature for $1 \mathrm{~h}$. The membrane was then incubated with the primary antibody (diluted according to the manufacturer's instructions) for $2 \mathrm{~h}$. Horseradish peroxidase-conjugated anti-rabbit or anti-mouse IgG was used as a secondary antibody. Immunoreactive proteins were visualized by chemiluminescence (ECL; Amersham, Arlington Heights, IL, USA).

Non-radioactive cytotoxicity assay. The oncolytic effect of adenoviruses was assessed using the CytoTox $96^{\circledR}$ Non-Radioactive Cytotoxicity Assay kit (Promega, Madison, WI, USA). This assay can quantitatively measure lactate dehydrogenase (LDH) levels, a stable cytosolic enzyme that is released upon cell lysis. After the various cancer cells were treated with 3-MA or rapamycin for $1 \mathrm{~h}$, they were infected with two types of oncolytic adenovirus of various MOIs and incubated for 3-7 days in 48-wells. A total of 50 $\mu \mathrm{l}$ of supernatant from each well was transferred onto a new 96-well flat-bottom (enzymatic assay) plate and $50 \mu \mathrm{l}$ of Reconstituted Substrate Mix were added to each well, followed by incubation 
A

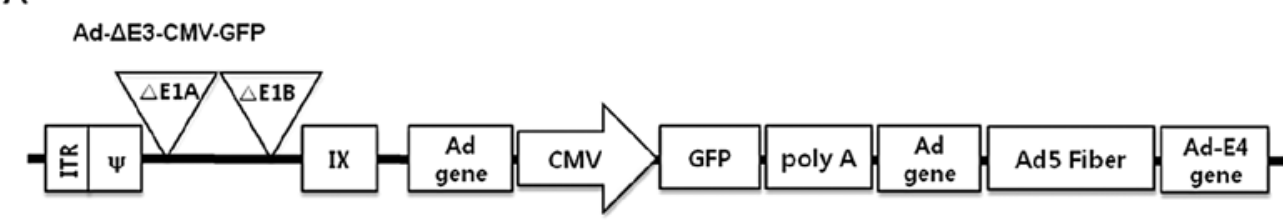

Ad- $\triangle$ E3-CMV-GFP-2374

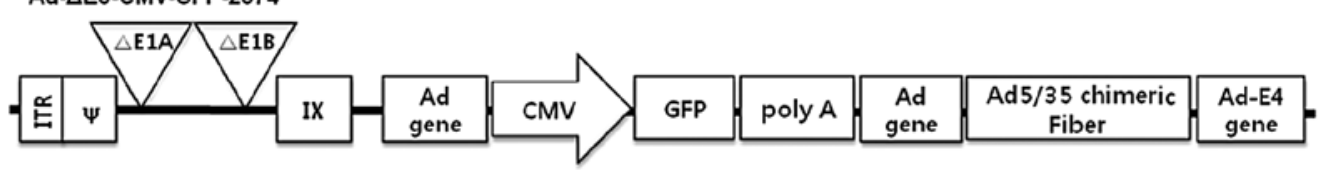

B

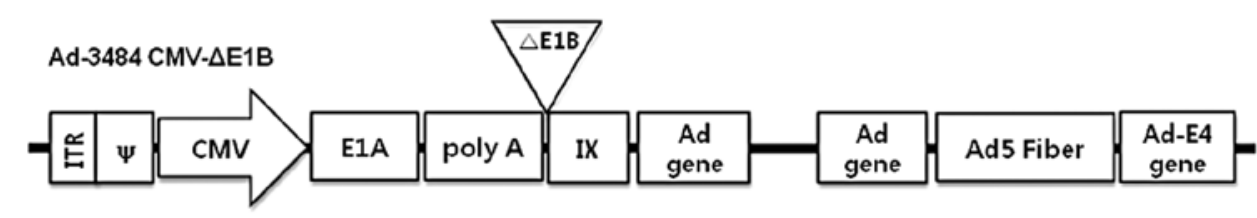

Ad-3484 CMV- $\Delta \mathrm{E} 1 \mathrm{~B}-2374$

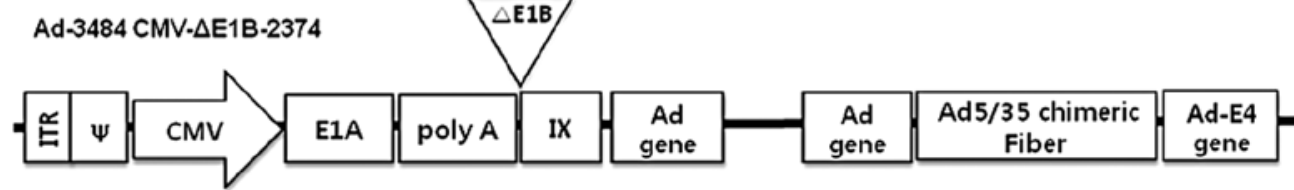

Figure 1. Schematic structure of adenoviral vectors. (A) Ad- $\Delta$ E3-CMV-GFP adenovirus: a replication-incompetent adenovirus used as the control, composed of the green fluorescence protein (GFP) gene, which is under the control of the cytomegalovirus (CMV) promoter and contains the adenovirus type 5 (Ad5) fiber gene at the E3 region. Ad- $\triangle \mathrm{E} 3-\mathrm{CMV}-\mathrm{GFP}-2374$ adenovirus contains chimeric type 5 and type 35 fiber proteins (Ad5/F35), but the remaining structure is identical to the control virus. (B) Ad-3484-CMV- $\Delta \mathrm{E} 1 \mathrm{~B}$ adenovirus is a replication-competent adenovirus used as the control and contains the E1A gene controlled by the CMV promoter but lacks the E1B region. Ad-3484-CMV- $\Delta$ E1B-2374 adenovirus is a replication-competent adenovirus, containing chimeric type 5 and type 35 fiber proteins, but the remaining structure is identical to the control virus.

for $30 \mathrm{~min}$ at room temperature in the dark. After $30 \mathrm{~min}, 50 \mu \mathrm{l}$ of stop solution were added to each well and the absorbance was examined at $490 \mathrm{~nm}$ within $1 \mathrm{~h}$, using an ELISA plate reader. For LDH positive control, lysis solution (10X) was added to the control cells and the lysed cells were incubated for $45 \mathrm{~min}$, after which time the plate was centrifuged at $250 \mathrm{x}$ g for $4 \mathrm{~min}$. Following centrifugation, $50 \mu \mathrm{l}$ of supernatant were transferred to an enzymatic assay plate for LDH assay, as described above.

Statistical analysis. Data are presented as the means \pm SEM of replicate samples, as described in the figure legends. The Student's $t$-test was used to compare two different groups. A value of $\mathrm{p}<0.05$ was considered to indicate a statistically significant difference.

\section{Results}

Transduction efficiency of Ad5/F35 in human cancer cell lines. The infectivity of Ad5/F35 was expected to be higher than that of Ad5 in the cancer cell lines expressing CAR or CD46. We first examined the transduction efficiency of GFP-expressing Ad5/F35 in a variety of human cancer cell lines to identify the types of cancer cells that may be suitable targets for chimeric Ad5/F35. Two types of adenoviruses that were replication-defective or tumor-selective and replication-competent were produced and each type exhibited an alteration in the fiber region. Their detailed structures are shown in Fig. 1. In general, melanoma cells are known to require high doses of adenovirus for sufficient gene transfer, due to the the paucity of CAR expression (19). However, it is unlikely that transduction efficiency correlates with a specific type of cancer cell. Compared to Ad5, chimeric Ad5/F35 did not exhibit higher transduction efficiency in SK-MEL-2, SK-MEL-3 and other cancer cell lines. To further investigate the effect of chimeric Ad5/F35 on gene transfer efficiency in melanoma cells (SK-MEL-2), chimeric Ad5/F35 adenovirus was infected at various MOIs. As shown in Fig. 2B, the transduction efficiency of Ad5/F35 at different MOIs was very similar to that of Ad5, which was consistent with the findings of Yu et al $(21,22)$.

Expression levels of CAR and CD46 in human cancer cell lines. Despite our expectations, the transduction efficiency of chimeric Ad5/F35 in most cancer cells was not enhanced compared to that of Ad5. Therefore, we investigated whether a correlation exists between the transduction efficiency of Ad5 or Ad5/F35 and CAR or CD46 expression levels in the plasma membrane of the cancer cells. We did not discover any direct correlation between the transduction efficiency and CD46 expression following the transfection of cancer cells with Ad5/F35. CAR and CD46 were highly expressed in the majority of the cancer cell lines, apart from the SK-MEL-3 cells (Fig. 3). This indicated that the infectivity of Ad5/F35 is not modified in relation to cell surface receptors. To confirm 


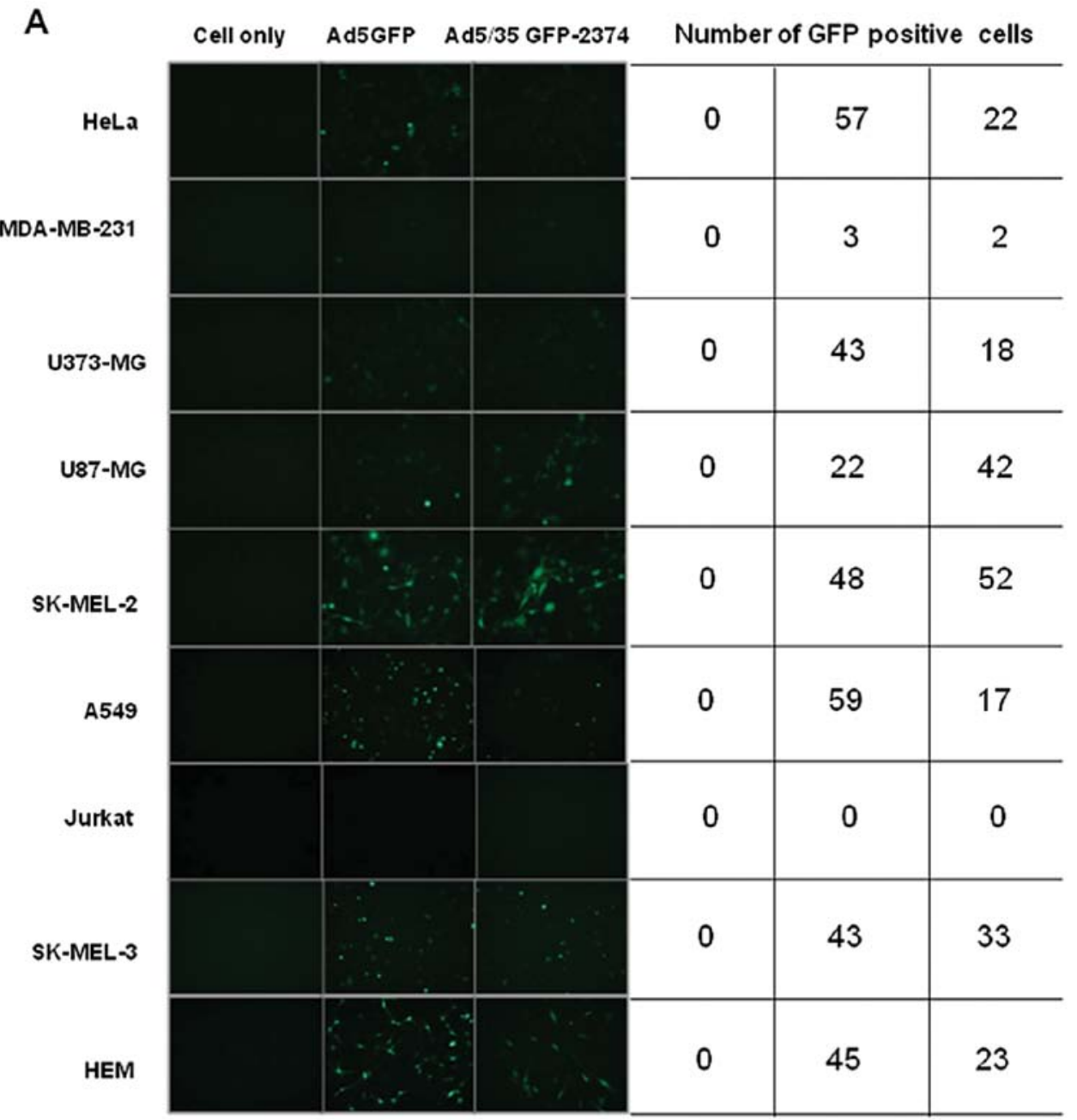

B

Ad-DE3-CMV-GFP

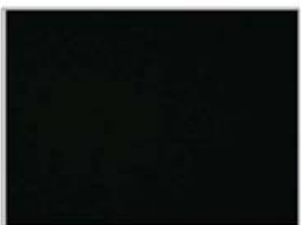

$0 \mathrm{MOI}$

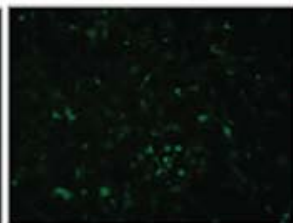

$5 \mathrm{MOI}$

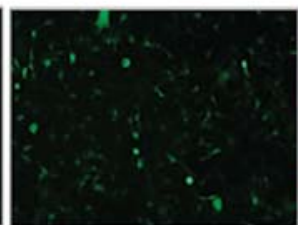

$10 \mathrm{MOI}$

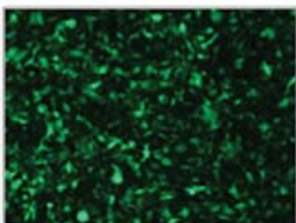

$50 \mathrm{MOI}$

\section{Ad- $\triangle$ E3-CMV-GFP-2374}

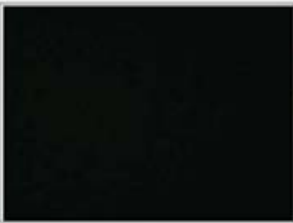

$0 \mathrm{MOI}$

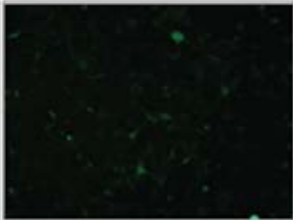

$5 \mathrm{MOI}$

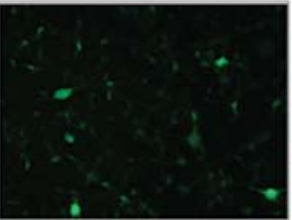

$10 \mathrm{MOI}$

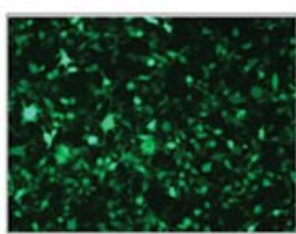

$50 \mathrm{MOI}$

Figure 2. Infectivity of GFP-expressing adenovirus type 5 and adenovirus Type 5/F35. (A) Various human cancer cells were infected with Ad5-GFP (control virus) and Ad-5/35-GFP (2374 virus) at a multiplicity of infection (MOI) of 20 . After 48 h, GFP expression was detected. For the quantitative transduction, GFP-positive cells were counted. (B) The SK-MEL-2 cell line was infected with the adenovirus at various MOIs. After 6 or $12 \mathrm{~h}$, viruses were removed and GFP expression was observed after $48 \mathrm{~h}$.

that the transduction efficiency of Ad5/F35 and the levels of $\mathrm{CD} 46$ were not the main factors responsible for optimal gene transfer, we investigated the possibility that autophagy facili- tates a faster induction of Ad5/F35 viral particle production compared to Ad5. According to recent studies, adenovirus has the ability to induce autophagy (36-39); therefore, autophagy 


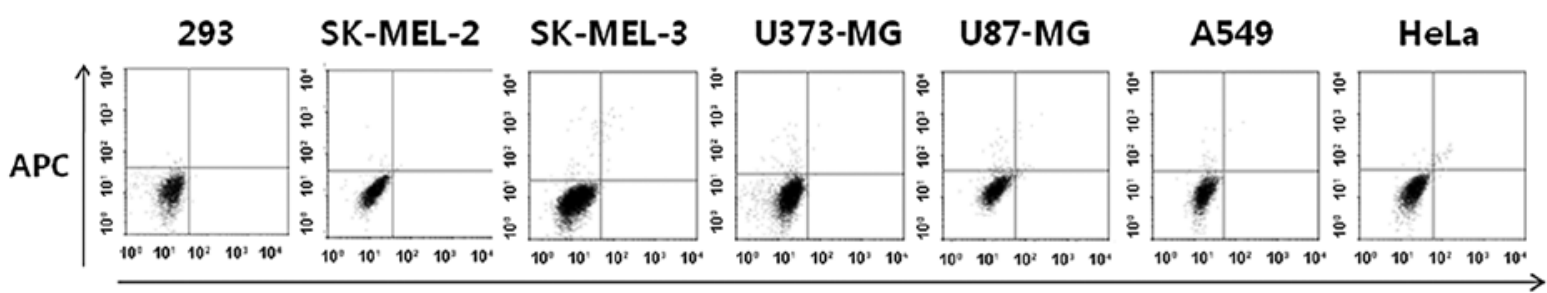

FITC

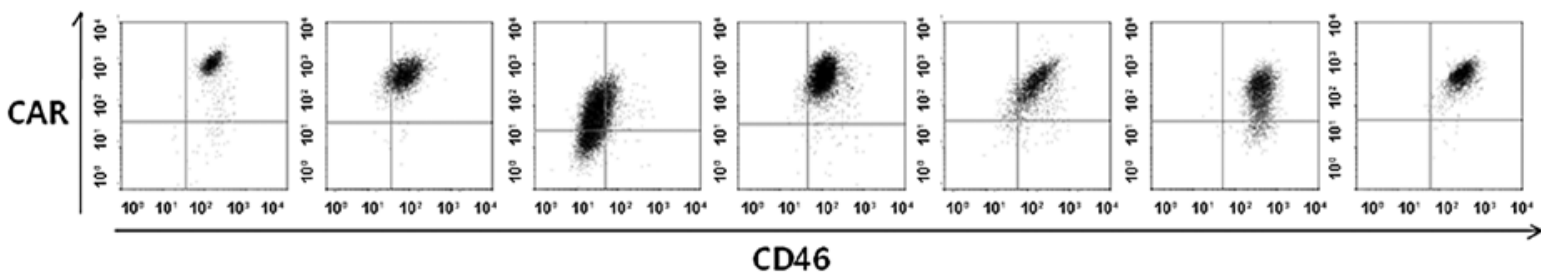

\begin{tabular}{l|ccccccc} 
& 293 & SK-MEL-2 & SK-MEL-3 & U373-MG & U87-MG & A549 & HeLa \\
\hline $\begin{array}{l}\text { CAR+/ } \\
\text { CD46- }\end{array}$ & $0 \%$ & $6.7 \%$ & $62.4 \%$ & $2 \%$ & $6.5 \%$ & $0 \%$ & $0 \%$ \\
\hline $\begin{array}{l}\text { CAR-/ } \\
\text { CD46+ }\end{array}$ & $2.9 \%$ & $0 \%$ & $0 \%$ & $0 \%$ & $2 \%$ & $9 \%$ & $0 \%$ \\
\hline $\begin{array}{l}\text { CAR+/ } \\
\text { CD46+ }\end{array}$ & $97 \%$ & $93 \%$ & $13 \%$ & $98 \%$ & $90 \%$ & $91 \%$ & $99 \%$ \\
\hline
\end{tabular}

Figure 3. Expression levels of coxsackievirus and adenovirus receptor (CAR) and CD46 in various cancer cell lines by flow cytometric analysis. Allophycocyanin (APC) single-positive cells express CAR, but not CD46, whereas FITC single-positive cells express CD46 only. The cells expressing CD46 and CAR stained double-positive for APC and FITC.
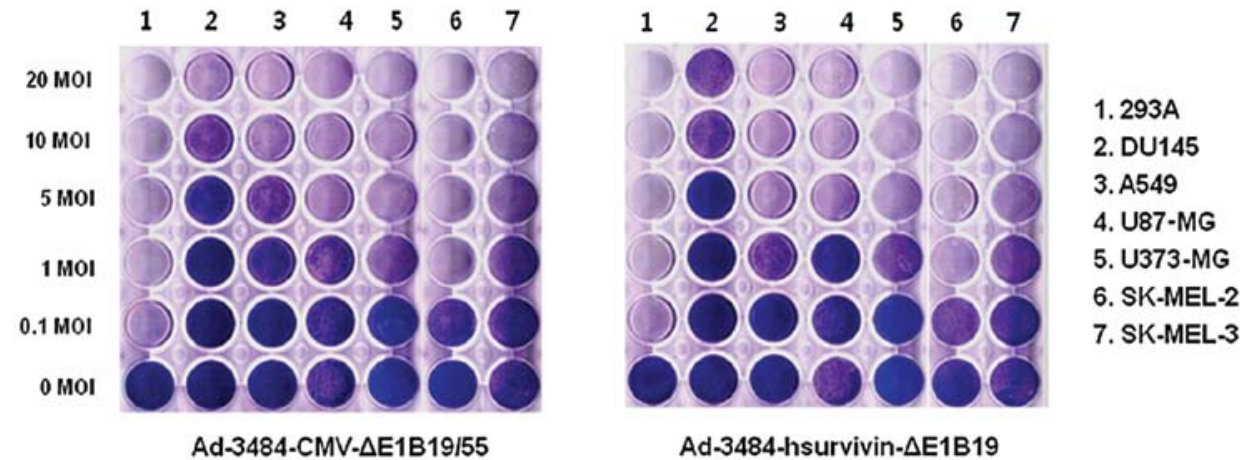

Figure 4. Comparison of the oncolytic activity induced by Ad-3484-CMV- $\Delta$ E1B19/55 with that of Ad-3484-hsurvivin- $\Delta$ E1B19. To compare the oncolytic activity induced by Ad-3484-CMV- $\Delta$ E1B19/55 with the one induced by Ad-3484-hsurvivin- $\Delta$ E1B19, cancer cells were infected with each virus at an MOI between 0.1 and 20. Immediately after the 293A cells infected with one of the viruses at an MOI of 0.1 exhibited complete cell lysis, all the remaining cells on the plate were fixed with $4 \%$ paraformaldehyde and stained with $0.5 \%$ crystal violet.

may be used to facilitate the release of viral progeny, promote adenovirus replication and improve oncolysis $(23,39)$.

Oncolytic activity induced by tumor-selective, replicationcompetent adenoviruses in vitro. To estimate the oncolytic activity induced by adenoviruses, various tumor cells were infected with two types of replication-competent adenovirus (Ad-3484-CMV- $\Delta$ E1B and Ad-3484-hsurvivin- $\Delta$ E1B19KD). For Ad-3484-CMV- $\Delta$ E1B, E1A gene expression was regulated by the CMV promoter and the entire E1B gene was deleted, whereas in the case of Ad-3484-hsurvivin- $\triangle$ E1B19KD, E1A expression was regulated by the human survivin promoter and only E1B55KD was expressed. The oncolytic activity induced by these viruses was very similar in all the cancer cells examined (Fig. 4), whereas no oncolytic activity was observed in normal cells (data not shown). In this study, we also compared the oncolytic activity induced by Ad-3484-CMV- $\Delta$ E1B with Ad5 fiber (control) with that of Ad-3484-CMV- $\Delta$ E1B-2374 with chimeric Ad5/F35 in various cancer cell lines (Fig. 5A). As previously mentioned, we also examined whether the autophagic condition is essential for oncolytic activity. For this experiment, rapamycin was used as an autophagy inducer and 3-MA, as well as wortmannin [phosphoinositide 3-kinase (PI3K) inhibitors], were used as autophagy inhibitors, based on their inhibitory effect 
A

$\begin{array}{llllllllllllllllllllllll}1 & 2 & 3 & 4 & 1 & 2 & 3 & 4 & 1 & 2 & 3 & 4 & 1 & 2 & 3 & 4 & 1 & 2 & 3 & 4 & 1 & 2 & 3 & 4\end{array}$

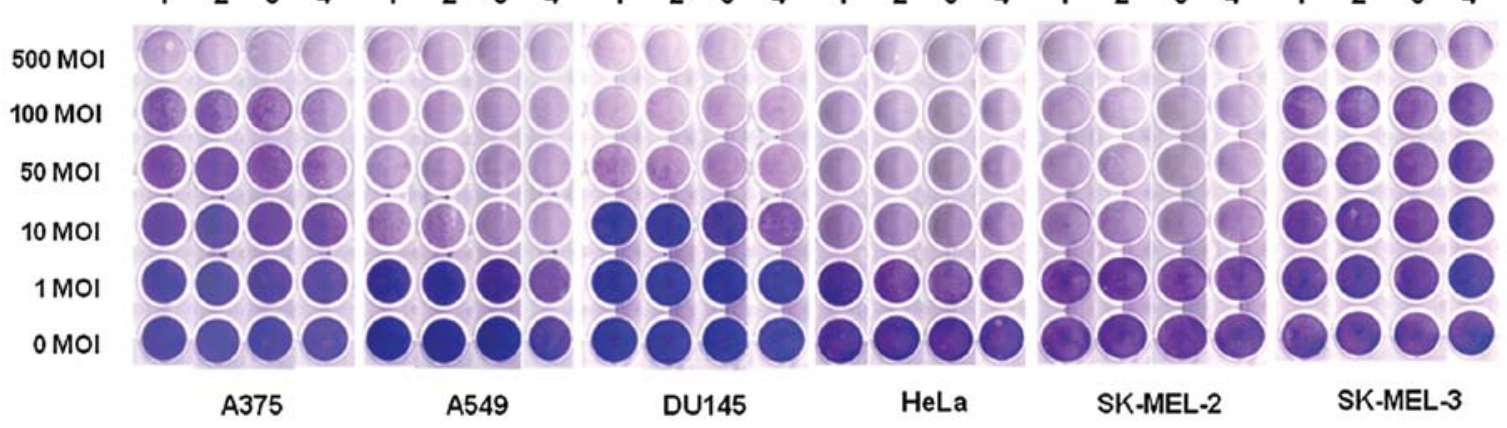

1. Ad-3484-CMV- $\triangle \mathrm{E} 1 \mathrm{~B}$

3. Ad-3484-CMV- $\triangle E 1 B-2374+3-M A(150 \mathrm{nM})$

2. Ad-3484-CMV- $\triangle \mathrm{E} 1 \mathrm{~B}-2374$

4. Ad-3484-CMV- $-\mathrm{E} 1 \mathrm{~B}-2374+$ rapamycin $(100 \mathrm{nM})$

B
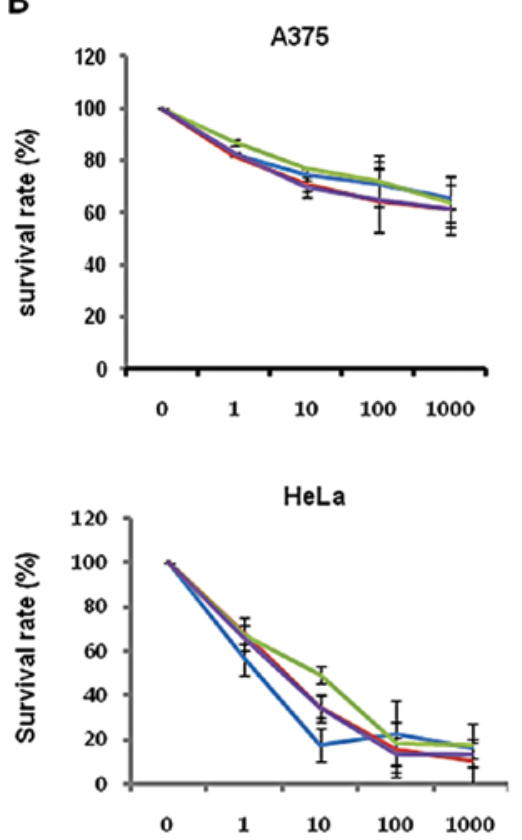

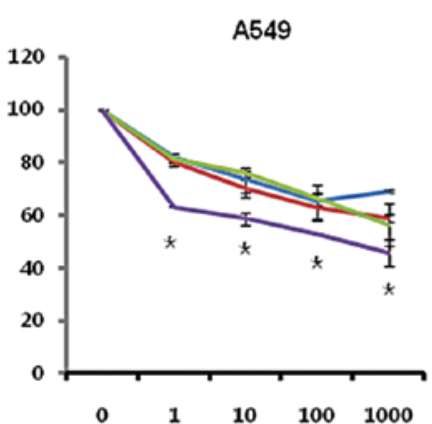

SK-MEL-2

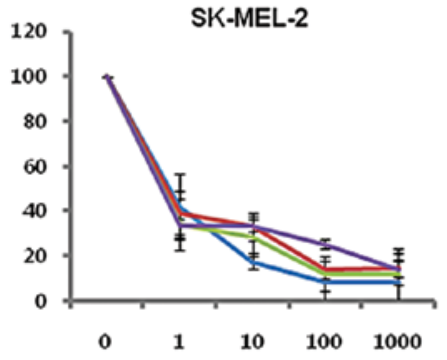

DU145

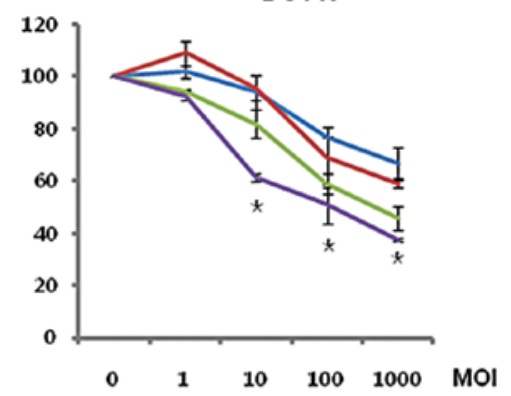

SK-MEL-3

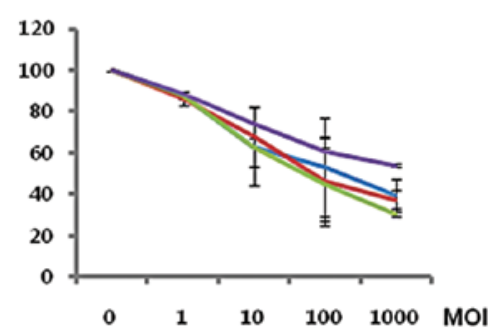

$-3484-2374-2374+3-M A-2374+$ rapamycin

C

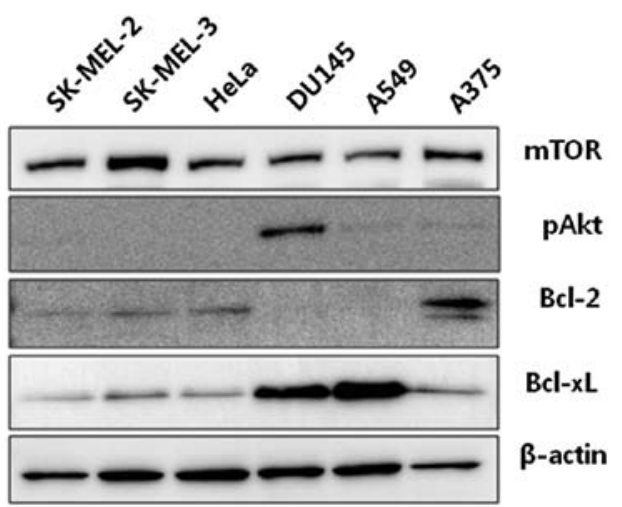

Figure 5. Comparison of the oncolytic activity induced by Ad-3484-CMV- $\Delta$ E1B to that induced by Ad-3484-CMV- $\Delta$ E1B-2374 and survival signals/doubling time of examined cancer cells. (A) To compare the oncolytic activities of Ad-3484-CMV- $\Delta$ E1B and Ad-3484-CMV- $\Delta$ E1B-2374, various cancer cells were infected with each virus at an MOI between 1 and 500, following pre-treatment with 3-MA $(150 \mathrm{nM})$ or rapamycin $(100 \mathrm{nM})$ for $1 \mathrm{~h}$. After seven days of infection (five days for HeLa and SK-MEL-2 cells), all the cells on the plate were fixed with $4 \%$ paraformaldehyde and stained with $0.5 \%$ crystal violet. (B) Various cancer cells were infected with each virus mentioned in (A) at an MOI between 1 and 1000, following pre-treatment with 3-MA (150 nM) or rapamycin (100 nM) for $1 \mathrm{~h}$. After seven days of infection (five days for HeLa and SK-MEL-2 cells), LDH cytotoxic assay was performed to determine the comparative potency of each treatment. 3484, Ad-3484-CMV- $\Delta$ E1B; 2374, Ad-3484-CMV- $\Delta$ E1B-2374; 2374 + 3-MA, Ad-3484-CMV- E1B-2374 + 3-MA; 2374 + rapamycin, Ad-3484-CMV- $\Delta$ E1B-2374 + rapamycin. Error bars represent standard errors from three separate experiments. The asterisks $(*)$ indicate a significant difference of $2374+$ rapamycin compared to 3484 (p<0.05). (C) Survivalrelated signals, such as mTOR, phosphorylated Akt, Bcl-2 and Bcl-xL, were examined in the cancer cells shown in (A). 
Table I. Doubling times of various cancer cell lines.

\section{Cell line}

Doubling time (h)

$\begin{array}{ll}\text { DU145 } & 16.2 \pm 6.2 \\ \text { A549 } & 21.7 \pm 6.1 \\ \text { HeLa } & 12.3 \pm 1.4 \\ \text { A375 } & 10.8 \pm 2.4 \\ \text { SK-MEL-3 } & 32.0 \pm 10 \\ \text { SK-MEL-2 } & 43.6 \pm 10\end{array}$

Cancer cells were plated onto 12 -well plates $\left(1.5 \times 10^{4}-5 \times 10^{4}\right.$ cells, depending on the cell type) and counted every $24 \mathrm{~h}$ for three days. The doubling time was calculated during the highest increase in cell number.

on class III PI3K activity, which is essential for the induction of autophagy (40). As shown in Fig. 5A, the oncolytic activity induced by chimeric Ad5/F35 was higher in DU145 and A549 cells following rapamycin treatment, but not in SK-MEL-2, SK-MEL-3, A375 and HeLa cells. To verify this comparative potency of the treatments, LDH cytotoxicity assay was performed. We discovered that the oncolytic activity induced by chimeric Ad5/F35 was higher in the DU145 and A549 cells following pre-treatment with rapamycin, by comparing the survival rates of the cells treated with rapamycin to those of the cells transfected with chimeric Ad5/F35 without rapamycin pre-treatment (DU145, 96 $\rightarrow 61 \%$ and A549, 76 $\rightarrow 59 \%$ ), at an MOI of 10 (Fig. 5B). We then aimed to determine the factor(s) that mainly control(s) the autophagy-enhanced oncolytic effect. We first examined the endogenous levels of several key molecules related to survival or anti-apoptosis, such as mTOR, phosphorylated Akt and Bcl-2 or Bcl-xL (Fig. 5C). As shown in Fig. 5C, DU145, A375 and A549 cells have higher survival potentials, whereas SK-MEL-2, SK-MEL-3 and HeLa cells have lower survival potentials. We also calculated the doubling time of each cell line in search of a correlation between oncolytic activity and proliferation rate (Table I). Based on our overall results, we discovered a correlation between oncolytic activity following rapamycin pre-treatment and survival potential. Accordingly, rapamycin-pre-treated DU145 cells exhibited the highest oncolytic activity. To confirm this finding, several other cancer cells with a higher survival potential were also examined using rapamycin, 3-MA and wortmannin, after narrowing down the range of MOIs. Our data indicated that Ad-3484CMV- $\Delta$ E1B-2374 with chimeric Ad5/F35 increased its oncolytic activity in the presence of rapamycin in DU145, A549,

\section{A-a}

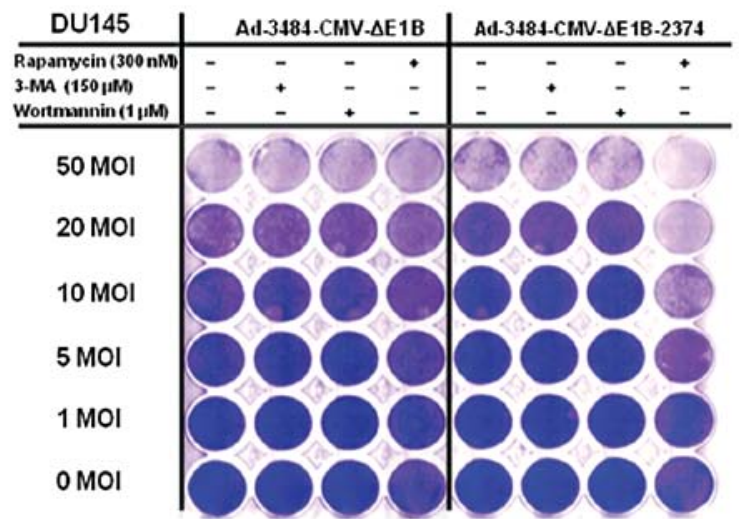
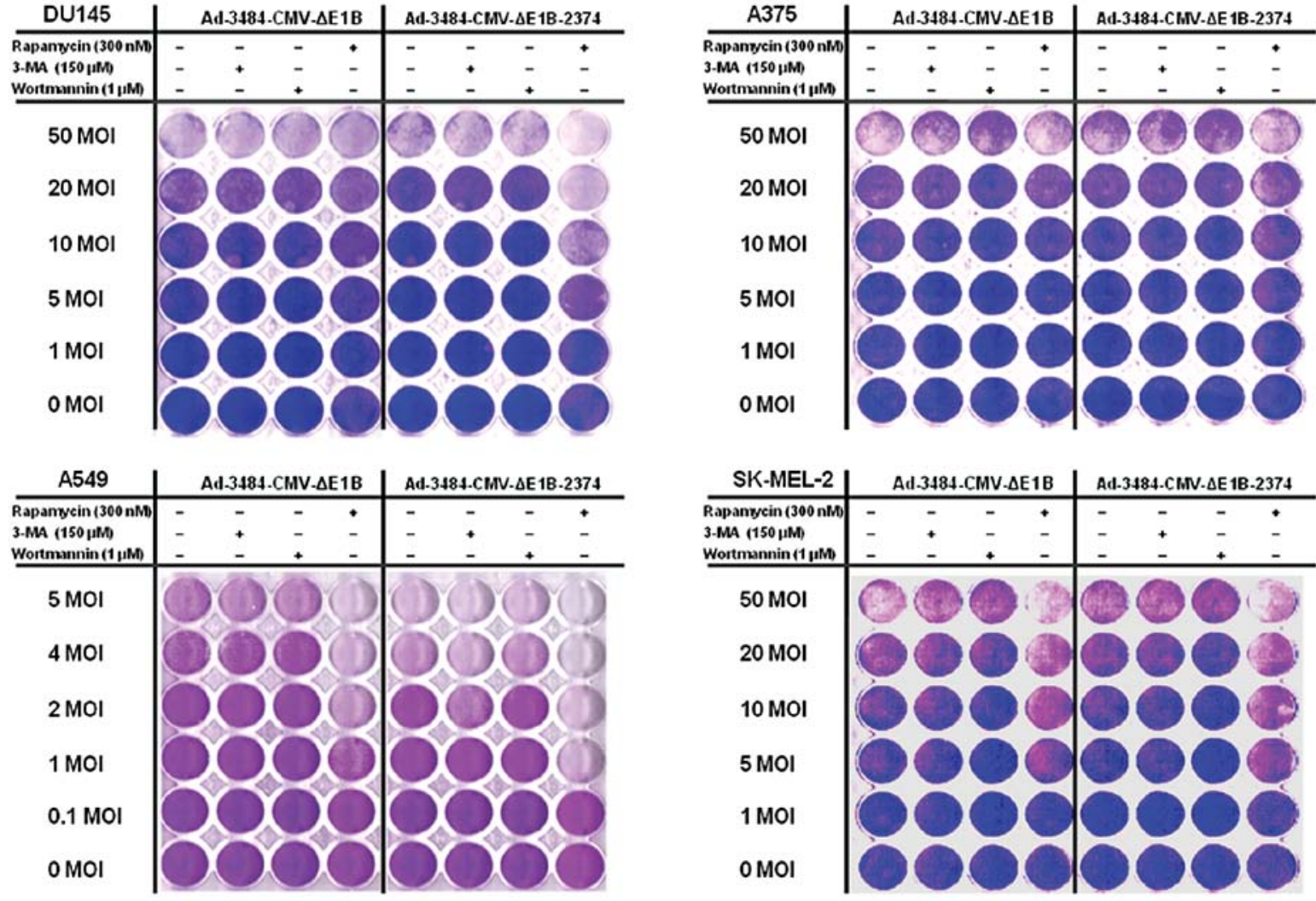

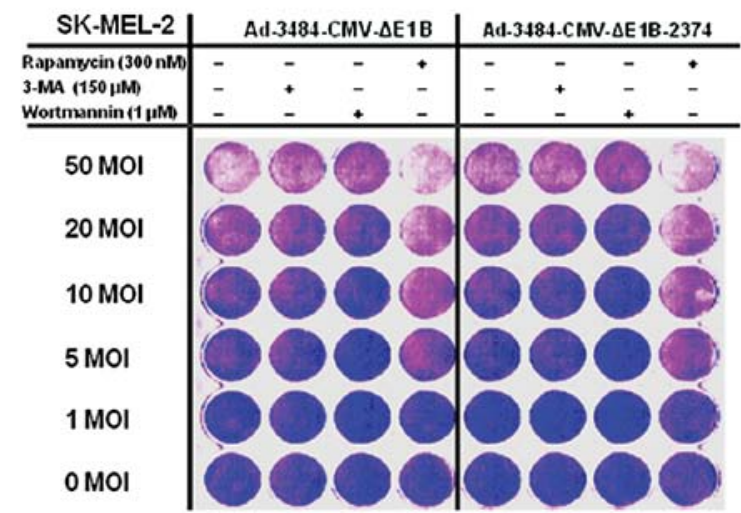

Figure 6. Comparison of oncolytic activity induced by Ad-3484-CMV- $\Delta$ E1B with that induced by Ad-3484-CMV- $\Delta$ E1B-2374 and survival signals/doubling time of examined cancer cells. (A) To compare the oncolytic activity induced by Ad-3484-CMV- $\Delta$ E1B with that of Ad-3484-CMV- $\Delta$ E1B-2374, cancer cells were infected with each virus at an MOI between 0.1 and 50, following pre-treatment with 3-MA (150 nM) or rapamycin (300 nM) or wortmannin $(1 \mu \mathrm{M})$ for $1 \mathrm{~h}$. After seven days of infection (five days for HeLa and SK-MEL-2 cells), all the cells on the plate were fixed with $4 \%$ paraformaldehyde and stained with $0.5 \%$ crystal violet. Left column: Lane 1, Ad-3484-CMV- $\Delta$ E1B; Lane 2, Ad-3484-CMV- $\Delta$ E1B + 3-MA (150 nM), Lane 3, Ad-3484-CMV- $\Delta$ E1B + wortmannin (1 $\mu$ M), Lane 4 , Ad-3484-CMV- $\Delta$ E1B + rapamycin (300 nM). Right column: Lane 1, Ad-3484-CMV- $\Delta$ E1B-2374; Lane 2, Ad-3484-CMV- $\Delta$ E1B-2374 + 3-MA (150 nM), Lane 3, Ad-3484-CMV- $\Delta$ E1B-2374 + wortmannin (1 $\mu$ M), Lane 4, Ad-3484-CMV- $\Delta$ E1B-2374 + rapamycin (300 nM). (a) DU145, A375, A549 and SK-MEL-2 cells . 
A-b

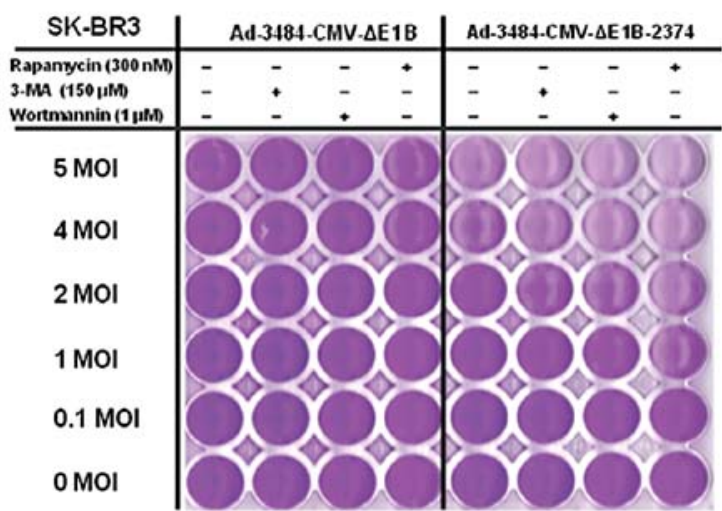

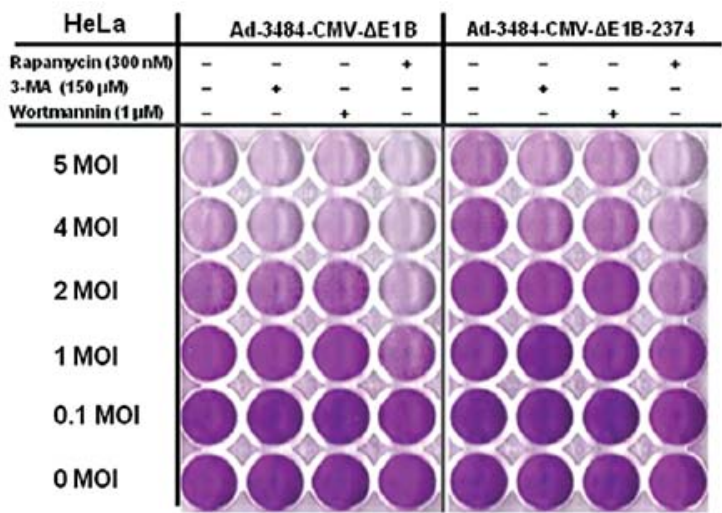

A-c

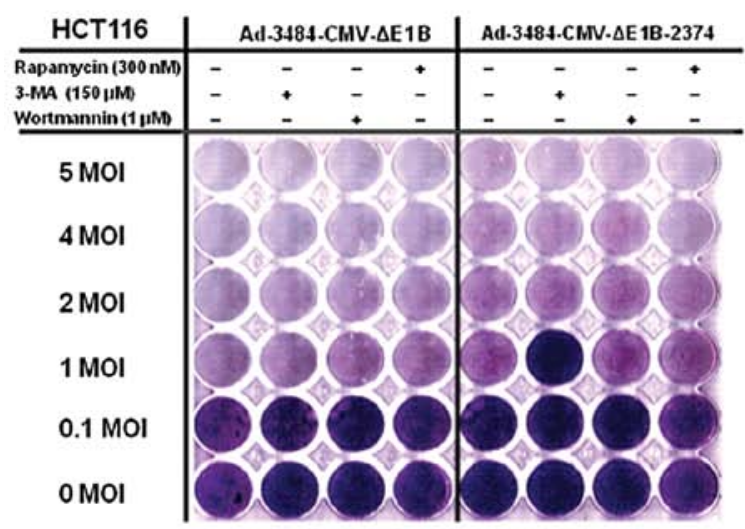

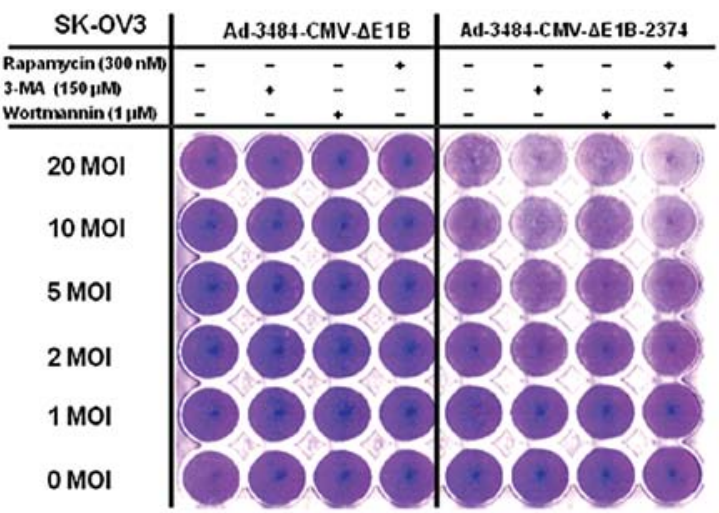

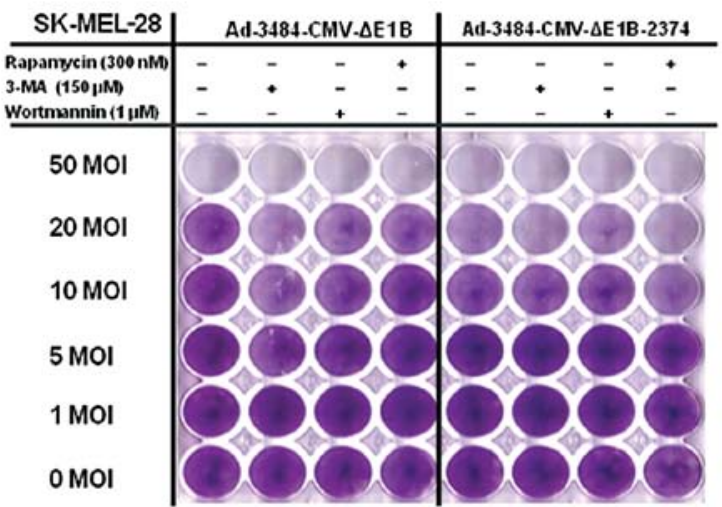

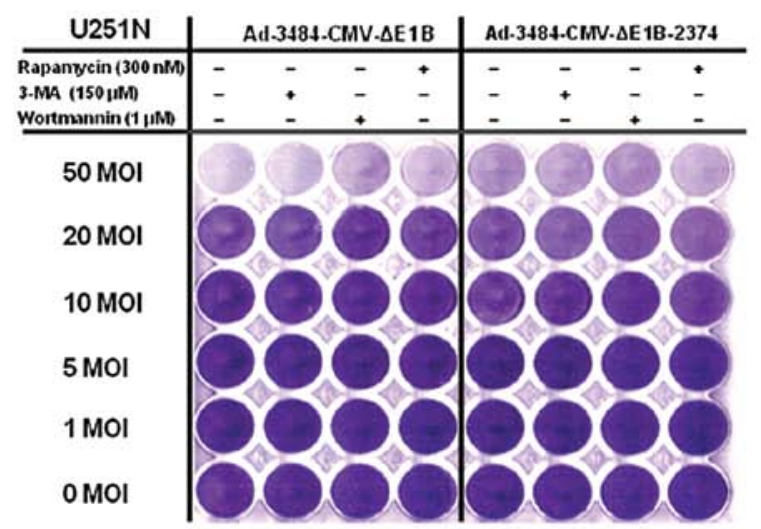

Figure 6. Continued. (b) SK-BR3, SK-OV3, HeLa and SK-MEL-28 cells. (c) HCT116 and U251N cells.

SK-MEL-28 and A375 cells, whereas no increase in oncolytic activity in the presence of rapamycin was observed in SK-MEL-2, U251N and HCT116 cells. SK-BR3 and SK-OV3 cells exhibited some oncolytic activity induced by these adenoviruses, irrespective of chemical treatment; however, the oncolytic effect of the Ad-3484-CMV- $\Delta \mathrm{E} 1 \mathrm{~B}-2374$ virus was less significant than that of the control virus on HeLa cells (Fig. 6A). As shown in Fig. 6A, the selective improvement in the oncolytic effect induced by Ad-3484-CMV- $\Delta$ E1B-2374 + rapamycin was also observed in DU145, A549, SK-MEL-28, SK-BR3 and SK-OV3 cells, as indicated by the survival rates (DU145, $69 \rightarrow 32 \%$; A549, 56 $\rightarrow 36 \%$; SK-MEL-28, 52 $\rightarrow 37 \%$; SK-BR3, 67 $\rightarrow 45 \%$ and SK-OV3, $83 \rightarrow 50 \%$ ), at $50 \mathrm{MOI}$. As regards the latter two cell lines (SK-BR3 and SK-OV3), the selectivity was only observed with Ad-3484-CMV- $\triangle \mathrm{E} 1 \mathrm{~B}-2374$, irrespective of the presence of inhibitors (Fig. 6A). Similar patterns of oncolytic activity were also observed by LDH cytotoxicity assay (Fig. 6B). The survival potential of each cell line was investigated (Fig. 6C) and the doubling time was estimated (Table II). However, as shown in Fig. 6D, the reduced oncolytic activity induced by Ad5/F35 in HeLa cells may be explained by the possibility that the cellular autophagic condition in HeLa cells is already established without an autophagy inducer, which results in less oncolytic activity induced by Ad5/F35 than by Ad5. Based on these results (Figs. 5 and 6, Tables I and II), we created a frequency diagram to demonstrate the correlation between 


\section{B-a}

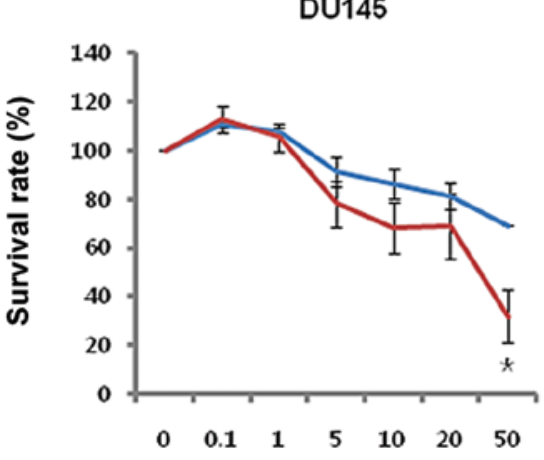

SK-MEL-2

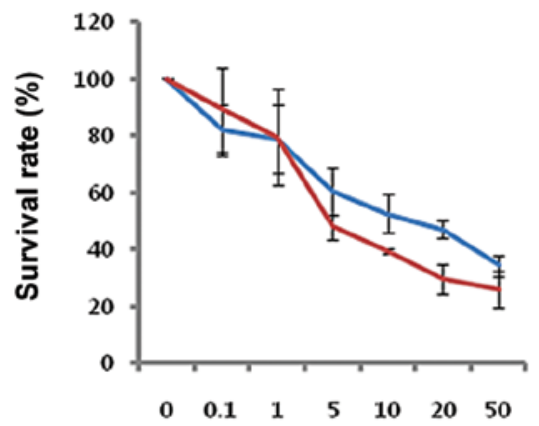

A375

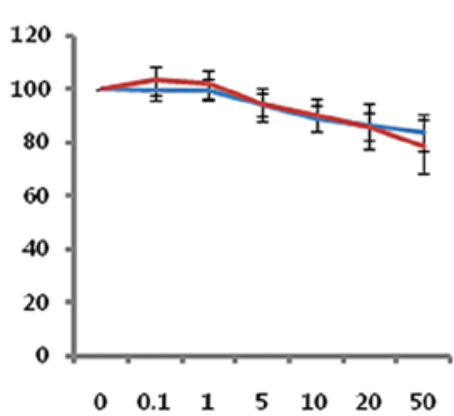

SK-BR 3

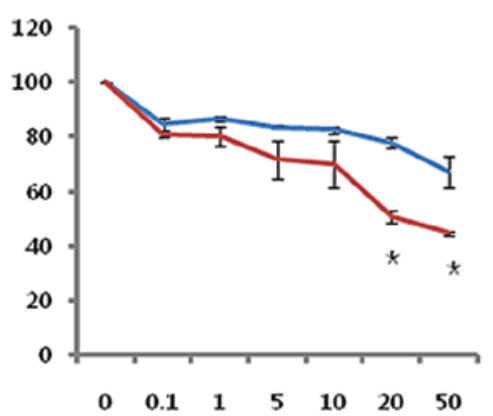

A549

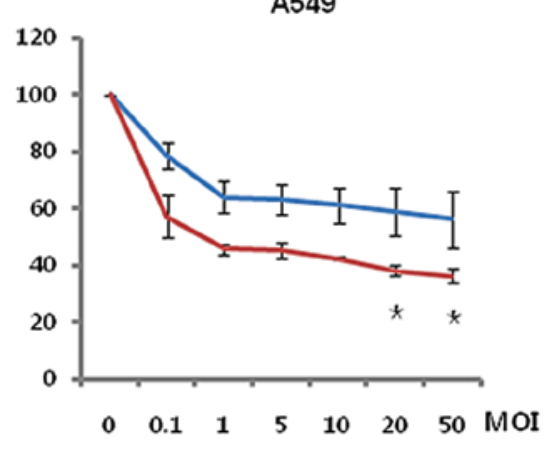

SK-OV 3

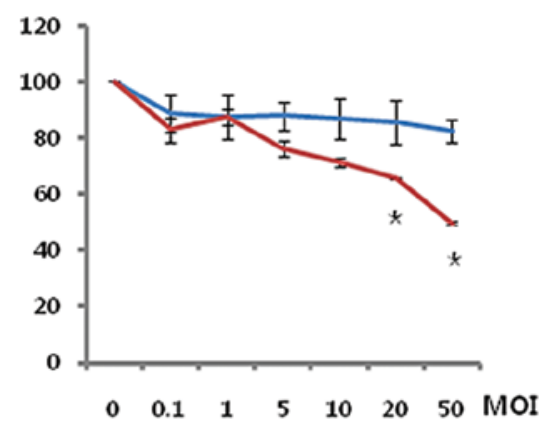

-3484 + rapamycin -2374 + rapamycin

\section{B-b}

HeLa

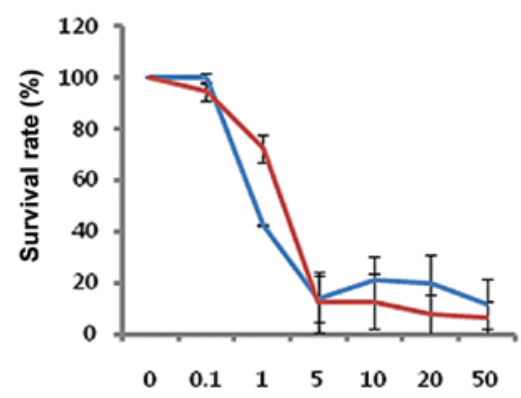

HCT116

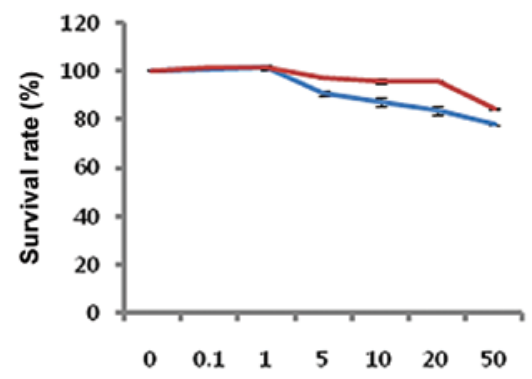

SK-MEL-28

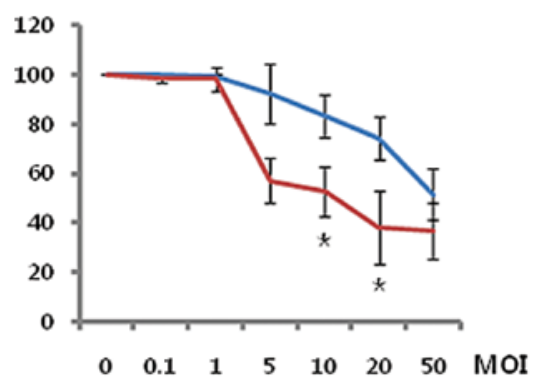

U251N

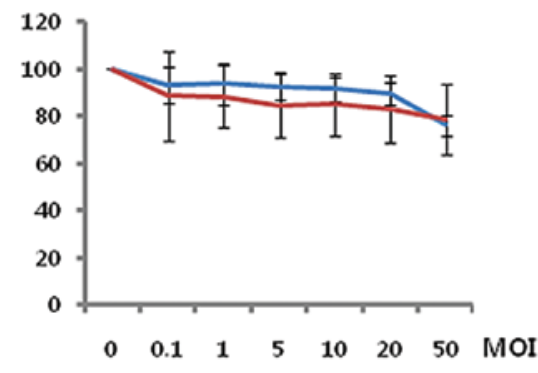

Figure 6. Continued. (B) Various cancer cells were infected with Ad-3484-CMV- $\Delta$ E1B or Ad-3484-CMV- $\Delta$ E1B-2374 at an MOI between 0.1 and 50, following pre-treatment with rapamycin $(300 \mathrm{nM}$ ) for $1 \mathrm{~h}$. After seven days of infection (five days for HeLa and SK-MEL-2 cells), LDH cytotoxic assay was performed to determine the comparative potency of each treatment. 3484 + rapamycin, Ad-3484-CMV- $\Delta \mathrm{E} 1 \mathrm{~B}+$ rapamycin (300 nM); 2374 + rapamycin, Ad-3484-CMV$\Delta$ E1B-2374 + rapamycin $(300 \mathrm{nM})$. Error bars represent standard errors from three separate experiments. The asterisks $(*)$ indicate a significant difference between 2374 + rapamycin and 3484 + rapamycin (p<0.05). (a) DU145, A375, A549, SK-MEL-2, SK-BR3 and SK-OV3 cells. (b) HeLa, SK-MEL-28, HCT116 and U251N cells. 

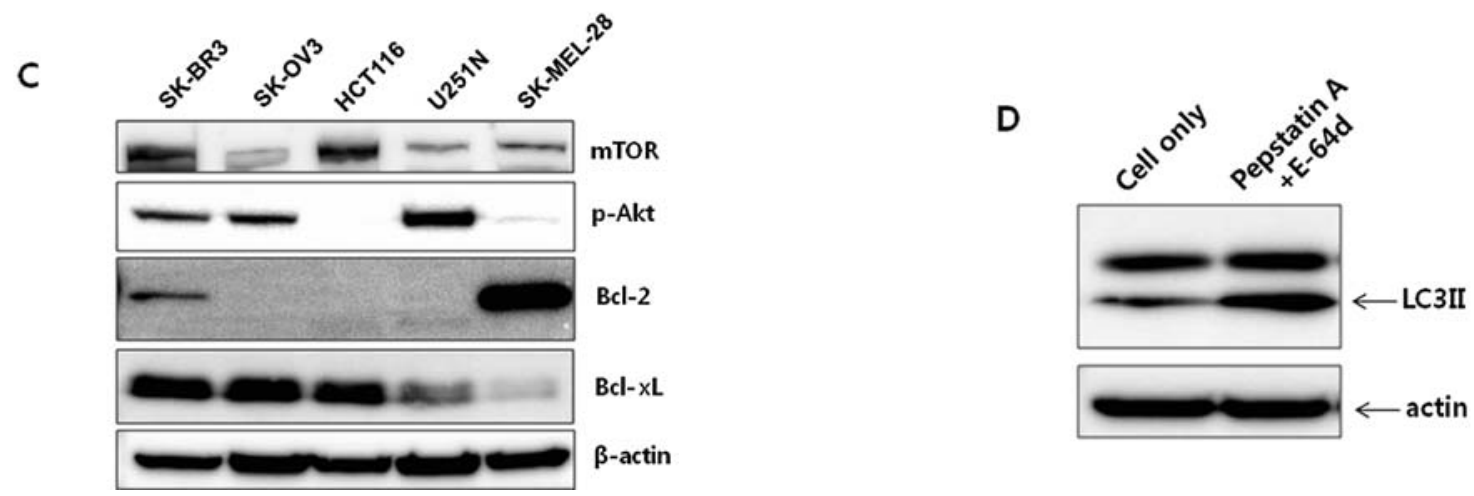

Figure 6. Continued. (C) Survival-related signals, such as mTOR, phosphorylated Akt, Bcl-2 and Bcl-xL, were examined in the cancer cells shown in (A). (D) LC3II conversion as a marker of autophagic activity was detected in the resting state of HeLa cells by pepstatin A $(20 \mu \mathrm{g} / \mathrm{ml})+\mathrm{E}-64 \mathrm{~d}(20 \mu \mathrm{g} / \mathrm{ml})$ for $4 \mathrm{~h}$.

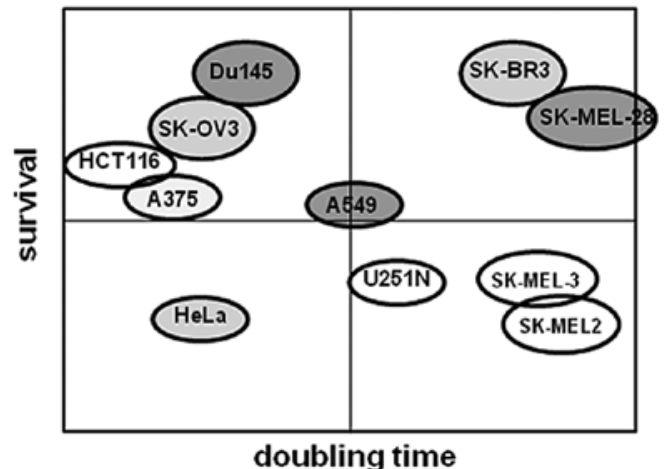

Figure 7. Correlation of the oncolytic activity induced by Ad-3484-CMV$\Delta \mathrm{E} 1 \mathrm{~B}-2374+$ rapamycin in cancer cells with a higher survival potential. DU145, A549 and SK-MEL-28 cells exhibited the most significant increase in oncolytic activity with Ad-3484-CMV- $\triangle$ E1B-2374 + rapamycin, compared to Ad-3484CMV- $\Delta$ E1B + rapamycin; A375 cells exhibited a less significant increase in oncolytic activity induced by Ad-3484-CMV- $\Delta$ E1B-2374 + rapamycin, compared to Ad-3484-CMV- $\Delta$ E1B + rapamycin; SK-OV3 and SK-BR3 cells exhibited the least significant increase in oncolytic activity induced by Ad-3484-CMV- $\Delta$ E1B-2374 + rapamycin, compared to Ad-3484-CMV$\triangle \mathrm{E} 1 \mathrm{~B}+$ rapamycin; HCT116, U251N, SK-MEL-2 and SK-MEL-3 cells exhibited no modifications in oncolytic activity; HeLa cells exhibited a reduction in oncolytic activity induced by Ad-3484-CMV- $\Delta$ E1B-2374 + rapamycin, compared to Ad-3484-CMV- $\Delta \mathrm{E} 1 \mathrm{~B}+$ rapamycin.

Table II. Doubling times of various cancer cell lines.

Cell line

SK-BR3

SK-OV3

HCT 116

$\mathrm{U} 251 \mathrm{~N}$

SK-MEL-28

Cancer cells were plated onto 12 -well plates $\left(1.5 \times 10^{4}-5 \times 10^{4}\right.$ cells, depending on the cell type) and counted every $24 \mathrm{~h}$ for three days. The doubling time was calculated during the highest increase in cell number.

survival or cell proliferation rate and oncolytic activity induced by $\mathrm{Ad} 5 / \mathrm{F} 35$ + autophagy inducer. As shown in Fig. 7, apart from the HCT116 cells, the majority of cancer cells exhibited higher survival potentials and increased oncolytic activities induced by $\mathrm{Ad} 5 / \mathrm{F} 35$ + autophagy inducer. However, we discovered that the cell proliferation rate was not related to oncolytic activity induced by Ad5/F35 + autophagy inducer. Taken together, our results suggest that rapamycin, being an autophagy inducer, exerts a pronounced effect on cancer cells with a higher survival potential.

\section{Discussion}

The aim of this study was to create a recombinant adenovirus with a modified fiber, in order to facilitate gene transfer in cancer cells and to overcome the poor tumor transduction efficiency of adenovirus serotype 5 (Ad5). Therefore, a chimeric adenovirus with an Ad5 fiber tail domain and a Ad35 fiber shaft and knob domain (Ad5/F35) was constructed, which is known to exhibit expanded tropism (17). However, as shown in Fig. 3, the exclusive expression of CD46 was not observed in all the cancer cell lines investigated; these results are consistent with the results shown in Fig. 2, where GFP-expressing Ad5/F35 did not show any increase in transduction efficiency. Another possible explanation of the relatively low transduction efficiency of Ad5/F35 may be due to the longer stay at endosomal/lysosomal compartments without endosomal escape for nuclear import, resulting in less efficient Ad5/F35-mediated gene transfer compared to that of Ad5 (41). We therefore investigated other means of enhancing Ad5/F35 transduction efficiency, on the basis that the CD46 receptor-promoted autophagy would result in the promotion of viral replication and oncolysis (23). The chimeric Ad5/F35 adenoviral vector exerted a more significant oncolytic effect on cancer cells with a higher survival potential under autophagic conditions generated by pre-treatment with rapamycin (an mTOR inhibitor) as an autophagy inducer (Figs. 5 and 6). Therefore, chimeric Ad5/F35 may be applicable in the treatment of cancer cells with a high survival potential. However, it is possible that rapamycin may increase the endosomal release of Ad5/F35, resulting in enhanced viral replication. This possibility is currently under investigation. Taken together, Ad5/F35-mediated higher gene transfer may be achieved by the combination of an oncolytic adenovirus with an autophagy inducer, such as rapamycin, or by incorporating genes that induce autophagy. 


\section{Acknowledgements}

This study was supported by the Industrial Strategic Technology Development program (10035562: Development of nucleic acid-based anticancer drugs overcoming immunotherapy resistance) funded by the Ministry of Knowledge Economy (MKE, Korea). This study was also supported by the Basic Science Research Program through the National Research Foundation of Korea (NRF) and funded by the Ministry of Education, Science and Technology (2012-0002108; grant to J.J.S.). S.Y.K. and S.K. were funded by the Brain Korea 21 Project for Medical Science, Yonsei University, College of Medicine, Seoul, Republic of Korea.

\section{References}

1. Fukazawa T, Matsuoka J, Yamatsuji T, Maeda Y, Durbin ML and Naomoto Y: Adenovirus-mediated cancer gene therapy and virotherapy (Review). Int J Mol Med 25: 3-10, 2010.

2. Sharma A, Tandon M, Bangari DS and Mittal SK: Adenoviral vector-based strategies for cancer therapy. Curr Drug Ther 4: 117-138, 2009.

3. Douglas JT: Adenoviral vectors for gene therapy. Mol Biotechnol 36: 71-80, 2007.

4. Russell SJ, Peng KW and Bell JC: Oncolytic virotherapy. Nature Biotech 30: 658-670, 2012.

5. Edukulla R, Woller N, Mundt B, et al: Antitumoral immune response by recruitment and expansion of dendritic cells in tumors infected with telomerase-dependent oncolytic viruses. Cancer Res 69: 1448-1458, 2009.

6. Rux JJ and Burnett RM: Adenovirus structure. Hum Gene Ther 15: 1167-1176, 2004.

7. Burnett RM: The structure of the adenovirus capsid. II. The packing symmetry of hexon and its implications for viral architecture. J Mol Biol 185: 125-143, 1985.

8. Wickham TJ, Segal DM, Roelvink PW, et al: Targeted adenovirus gene transfer to endothelial and smooth muscle cells by using bispecific antibodies. J Virol 70: 6831-6838, 1996.

9. Stevenson SC, Rollence M, Marshall-Neff J and McClelland A: Selective targeting of human cells by a chimeric adenovirus vector containing a modified fiber protein. J Virol 71: 4782-4790, 1997.

10. Zabner J, Freimuth P, Puga A, Fabrega A and Welsh MJ: Lack of high affinity fiber receptor activity explains the resistance of ciliated airway epithelia to adenovirus infection. J Clin Invest 100: 1144-1149, 1997.

11. Chillon M, Bosch A, Zabner J, et al: Group D adenoviruses infect primary central nervous system cells more efficiently than those from group C. J Virol 73: 2537-2540, 1999.

12. Tomko RP, Xu R and Philipson L: HCAR and MCAR: the human and mouse cellular receptors for subgroup $\mathrm{C}$ adenoviruses and group B coxsackieviruses. Proc Natl Acad Sci USA 94 3352-3356, 1997

13. Shayakhmetov DM, Papayannopoulou T, Stamatoyannopoulos G and Lieber A: Efficient gene transfer into human CD34(+) cells by a retargeted adenovirus vector. J Virol 74: 2567-2583, 2000.

14. Wickham TJ, Tzeng E, Shears LL II, et al: Increased in vitro and in vivo gene transfer by adenovirus vectors containing chimeric fiber proteins. J Virol 71: 8221-8229, 1997.

15. Wickham TJ, Lee GM, Titus JA, et al: Targeted adenovirusmediated gene delivery to T cells via CD3. J Virol 71: 7663-7669, 1997.

16. Okada Y, Okada N, Nakagawa S, et al: Fiber-mutant technique can augment gene transduction efficacy and anti-tumor effects against established murine melanoma by cytokine-gene therapy using adenovirus vectors. Cancer Lett 177: 57-63, 2002.

17. Mizuguchi $\mathrm{H}$ and Hayakawa $\mathrm{T}$ : Adenovirus vectors containing chimeric type 5 and type 35 fiber proteins exhibit altered and expanded tropism and increase the size limit of foreign genes. Gene 285: 69-77, 2002.

18. Joubert PE, Meiffren G, Grégoire IP, et al: Autophagy induction by the pathogen receptor CD46. Cell Host Microbe 6: 354-366, 2009.
19. Hemmi S, Geertsen R, Mezzacasa A, Peter I and Dummer R: The presence of human coxsackievirus and adenovirus receptor is associated with efficient adenovirus-mediated transgene expression in human melanoma cell cultures. Hum Gene Ther 9: 2363-2373, 1998.

20. Toyoda E, Doi R, Kami K, et al: Adenovirus vectors with chimeric type 5 and 35 fiber proteins exhibit enhanced transfection of human pancreatic cancer cells. Int J Oncol 33: 1141-1147, 2008.

21. Yu L, Shimozato O, Li Q, et al: Adenovirus type 5 substituted with type 11 or 35 fiber structure increases its infectivity to human cells enabling dual gene transfer in CD46-dependent and -independent manners. Anticancer Res 27: 2311-2316, 2007.

22. Yu L, Takenobu H, Shimozato O, et al: Increased infectivity of adenovirus type 5 bearing type 11 or type 35 fibers to human esophageal and oral carcinoma cells. Oncol Rep 14: 831-835, 2005.

23. Rodriguez-Rocha H, Gomez-Gutierrez JG, Garcia-Garcia A, et al: Adenoviruses induce autophagy to promote virus replication and oncolysis. Virology 416: 9-15, 2011.

24. Abounit K, Scarabelli TM and McCauley RB: Autophagy in mammalian cells. World J Biol Chem 3: 1-6, 2012.

25. Noda T and Ohsumi Y: Tor, a phosphatidylinositol kinase homologue, controls autophagy in yeast. J Biol Chem 273: 3963-3966, 1998 .

26. Asnaghi L, Bruno P, Priulla M and Nicolin A: mTOR: a protein kinase switching between life and death. Pharmacol Res 50: 545-549, 2004

27. Calastretti A, Rancati F, Ceriani MC, Asnaghi L, Canti G and Nicolin A: Rapamycin increases the cellular concentration of the BCL-2 protein and exerts an anti-apoptotic effect. Eur J Cancer 37: 2121-2128, 2001.

28. Ravikumar B,BergerZ, VacherC, O'Kane CJ and Rubinsztein DC: Rapamycin pre-treatment protects against apoptosis. Hum Mol Genet 15: 1209-1216, 2006

29. Everts B and van der Poel HG: Replication-selective oncolytic viruses in the treatment of cancer. Cancer Gene Ther 12: 141-161, 2005.

30. Guo ZS, Thorne SH and Bartlett DL: Oncolytic virotherapy: molecular targets in tumor-selective replication and carrier cell-mediated delivery of oncolytic viruses. Biochim Biophys Acta 1785: 217-231, 2008.

31. Wu L, Johnson M and Sato M: Transcriptionally targeted gene therapy to detect and treat cancer. Trends Mol Med 9: 421-429, 2003.

32. Diepgen TL and Mahler V: The epidemiology of skin cancer. Br J Dermatol 146 (Suppl 61): 1-6, 2002.

33. Lu B, Makhija SK, Nettelbeck DM, et al: Evaluation of tumorspecific promoter activities in melanoma. Gene Ther 12: 330-338, 2005.

34. Yun CO, Cho EA, Song JJ, et al: dl-VSVG-LacZ, a vesicular stomatitis virus glycoprotein epitope-incorporated adenovirus, exhibits marked enhancement in gene transduction efficiency. Hum Gene Ther 14: 1643-1652, 2003.

35. Yun CO, Kim E, Koo T, Kim H, Lee YS and Kim JH: ADP-overexpressing adenovirus elicits enhanced cytopathic effect by induction of apoptosis. Cancer Gene Ther 12: 61-71, 2005.

36. Baird SK, Aerts JL, Eddaoudi A, Lockley M, Lemoine NR and McNeish IA: Oncolytic adenoviral mutants induce a novel mode of programmed cell death in ovarian cancer. Oncogene 27: 3081-3090, 2008.

37. Ito $\mathrm{H}$, Aoki $\mathrm{H}$, Kühnel F, et al: Autophagic cell death of malignant glioma cells induced by a conditionally replicating adenovirus. J Natl Cancer Inst 98: 625-636, 2006.

38. Jiang $\mathrm{H}$, Gomez-Manzano $\mathrm{C}$, Aoki $\mathrm{H}$, et al: Examination of the therapeutic potential of Delta-24-RGD in brain tumor stem cells: role of autophagic cell death. J Natl Cancer Inst 99: 1410-1414, 2007.

39. Jiang H, White EJ, Gomez-Manzano C and Fueyo J: Adenovirus's last trick: you say lysis, we say autophagy. Autophagy 4: 118-120, 2008.

40. Wu YT, Tan HL, Shui G, et al: Dual role of 3-methyladenine in modulation of autophagy via different temporal patterns of inhibition on class I and III phosphoinositide 3-kinase. J Biol Chem 285: 10850-10861, 2010.

41. Shayakhmetov DM, Li ZY, Ternovoi V, Gaggar A, Gharwan H and Lieber A: The interaction between the fiber knob domain and the cellular attachment receptor determines the intracellular trafficking route of adenoviruses. J Virol 77: 3712-3723, 2003. 